\title{
Design Of The Approximation Function of a Pedometer based on Artificial Neural Network for the Healthy Life Style Promotion in Diabetic Patients
}

\author{
A. Vega-Corona, M. Zárate-Banda, J.M. Barrón-Adame, R.A. Martínez-Celorio \\ Universidad de Guanajuato \\ Lascuráin de Retana No. 5 Centro \\ Guanajuato, Gto. México. \\ tono@salamanca.ugto.mx \\ D. Andina \\ Universidad Politécnica de Madrid \\ Av. Complutense No. 30. Ciudad Universitaria \\ Madrid (España). \\ d.andina@upm.es
}

\begin{abstract}
The present study describes the design of an Artificial Neural Network to synthesize the Approximation Function of a Pedometer for the Healthy Life Style Promotion. Experimentally, the approximation function is synthesized using three basic digital pedometers of low cost, these pedometers were calibrated with an advanced pedometer that calculates calories consumed and computes distance travelled with personal stride input. The synthesized approximation function by means of the designed neural network will allow to reply the calibration experiment for multiple patients with Diabetes Mellitus in Healthy Life Style promotion programs. Artificial Neural Networks have been developed for a wide variety of computational problems in cognition, pattern recognition, and decision making. The Healthy Life Style refer to adequate nutrient ingest, physical activity, time to rest, stress control, and a high self-esteem. The pedometer is a technological device that helps to control the physical activity in the diabetic patient. A brief description of the Artificial Neural Network designed to synthesize the Approximation Function, the obtained Artificial Neural Network structure and results in the Approximation Function synthesis for three patients are presented. The advantages and disadvantages of the method are discussed and our conclusions are presented.
\end{abstract}

keywords: Artificial Neural Networks, Pedometer, Approximation Function, Diabetes Mellitus, Healthy Life Style.

\section{Introduction}

In many research areas and in daily life there are present phenomena that have a multivariate order, it means, they can not be explain considering one variable. This can be observed in clinical cases and the Healthy Life Style $(H L S)$ definition is not the exception. Every day, diabetic patients face the challenge of keeping a $H L S$, and on it depends their well-being. The treatment guidelines is oriented to provide insulin and hypoglycemic [3], it is based on calculating the corporal weight of the patient $W_{i}$, the calories ingested $C_{i}$ and the physical activity $G_{i}$, producing a value determined by the glucose measurement in blood or urine, establishing the necessary insulin level $I_{i}$. We can express these parameters as a multi-variable function: $I_{i}=f\left(W_{i}, C_{i}, G_{i}\right)$. The multi-variability, the patient tolerance and response to the different kinds of insulin and hypoglycemic making the treatment complex. Due to some alteration in the habitual behavior of the patient, the established parameters are not always resolutory for personal treatment; for example, the calorific ingest in a social event, a day with big physical activity, or some situation in which the stress level increases the patient response. The established insulin program can be not the adequate for those days. In general, the health services organize the visit to a specialist which is a tiring process. First, the patient goes to the family doctor, who determines if the patient should or should not attend the specialist, while the time elapses and the patient could be unbalanced for a period of 2 or 3 weeks, if this becomes recurrent it predisposes to the early diabetes complications. It also exists other support service to the diabetic patients 
treatment as the self-help groups where the health professionals have the responsibility of monitoring the group and their activities. Not only, to participate in early stages in these groups helping the patient to acquire and to maintain the habit for a $H L S$. Despite the therapeutic objective of maintaining the patient's independence and to modify the least possible their $H L S$.

For 10 years, the health professionals have promote the use of auxiliar instruments in the diabetic treatment, like glucometers, pedometers, etc. It is determined by the cost, complexity and handling, the use of the technology and its instruments have not had a satisfactory acceptance, however, we can say that the acceptance is good. During the practice of diabetic patient's care and programs to encourage the self-care like strategy for a $H L S$. The evaluation of devices or instruments (pedometers) show a great variety available in the market; some are more accurate than others, and others more complete but also complex and expensive. It allows to recover information and to verify the behavior of the patient's physical activity. Then, we have observed how the appropriate use of the pedometer contributes to provide appropriate information to determine the personalized treatment. Considering that in Mexico diabetic patients are increasing every day. The human resources for health attention are insufficient and the market will provide different Pedometer and Glucometer models. Health professionals consider the following questions: Is there a reliable way to integrate the obtained information from Pedometers and Glucometers to modify or to personalize the diabetic patient's treatment?, is it possible without any doubt to guarantee a sure treatment for the patient?. This research contributes to generate ad-hoc instruments to the patient's necessities and offer as an alternative to the health professionals in order to interpret the derived information of the self-care and responds to the established questions.

In the complex problem solutions as engineering, biology, communications and health [5], the Artificial Intelligence $(A I)$ application is a widely used alternative. The Artificial Neural Networks $(A N N)$ is one of the $A I$ techniques that is used to solve complex problems. Neural networks have the characteristic to function as a Black-Box that transforms an input vector of $m$-dimensional space to an output vector in $n-$ dimensional space. Table 1 shows a multi - dimensional example in the clinical case when two symptoms are presented in a patient that can derive in four possible causes. However, if the symptomatic variables increases it is probable that the diagnosis complicates, and requires a second opinion.

An output function $Y_{i}$ is shown in table 1, it can take four possible values in function of the combination $(X 1, X 2)$,

\begin{tabular}{|c|c|c|}
\hline Cough $\left(X_{1}\right)$ & Headache $\left(X_{2}\right)$ & Diagnose $\left(Y_{i}\right)$ \\
\hline 0 & 0 & Healthy \\
\hline 0 & 1 & Pneumonia \\
\hline 1 & 0 & Flu \\
\hline 1 & 1 & Meningitis \\
\hline
\end{tabular}

Table 1. Example of the dimensionality in a health problem.

consequently it defines the function as $Y_{i}=f\left(X_{1}, X_{2}\right)$, where $\left(Y_{i}, X_{1}, X_{2}\right) \in\{0,1\}$. An $A N N$ can learn the $Y_{i}$ function and to solve the diagnostic problem described before. Actually, we want the $A N N$ acquires the knowledge of the diagnostic, hence the importance of this kind of $A I$ tool.

\section{Objectives}

In this research, the general objective is to design an $A N N$ to synthesize the $A F$ of a Pedometer that relates quantity of steps versus calorific burn for the HLS Promotion in Diabetic Patients

\subsection{Specific objectives}

1. Respectively, to build and to define the curve and the $A F$ of the calories burnt in function of number of steps for each person.

2. To design a Feed Forward Neural Network (FFNN), and to train under the Backpropagation algorithm to learn the $A F$.

3. To evaluate the $A F$ using the Minimum Square Error (MSE).

\section{Methodology}

\subsection{Material}

- In this research, two pedometers of the manufacturing Advanced Pendulum Design ${ }^{T M}$ were used, one economic model of the Series 330 (PT1) and other advanced of the Series 350 (PT2).

- The Matlab Reread 14 (Mathworks Inc.) was used as development software.

- "Walking Advantage 1" manuals of sportline were used.

\footnotetext{
${ }^{1}$ http://www.cialtia.com.mx
} 


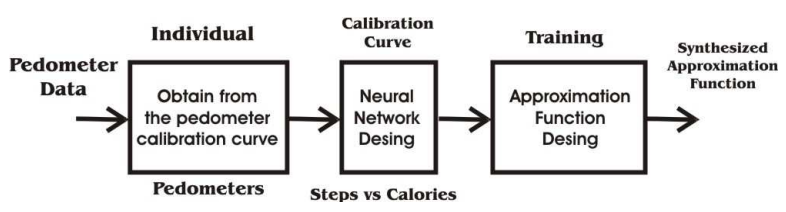

Figure 1. Implemented methodology in order to design the sintetized Approximation Function of the pedometer.

\section{Methods}

To establish the $(A F)$ construction method of the pedometer figure 1 was followed. It shows the sequence to build the AF from the characteristic curve of the pedometer.

\subsection{Obtaining the Calibration Curve of the Pedometers}

The first step to synthesize the $A F$ is the construction of the calibration curve of the pedometers. The calibration curve includes calories burn versus the number of steps for each person. In this stage, it was necessary to accomplish the calibration procedure of the mensuration instruments. For the calibration two pedometers were used defined as $P T 1$ and PT2. The PT1 (SPD330) is a basic pedometer which only counts steps, the PT2 (SPD350) is a pedometer that counts steps, calculates distance, calories burn and consequently is considered as the calibration pattern. The achieved calibration procedure making steps mensurations versus calories burnt from three different persons of weight and stride size, which are necessary for the pedometer configuration (See table 2). Configured the PT1 and PT2 pedometers, 10 measures between $35 \mathrm{~m}$ and $350 \mathrm{~m}$. (35m, $70 \mathrm{~m}, 105 \mathrm{~m}, 140 \mathrm{~m}, 175 \mathrm{~m}, 210 \mathrm{~m}, 245 \mathrm{~m}, 280 \mathrm{~m}, 315 \mathrm{~m}$ and $370 \mathrm{~m}$ ) were measure for each person. With PT2 the calories burnt were measured. in order to extract the characteristic curve in each corresponding block, each measurement in both pedometers were averaged and were extracted mean value and standard deviation.

\begin{tabular}{|c|c|c|}
\hline Person & Weight $(\mathrm{Kg})$ & Stride $(\mathrm{cm})$ \\
\hline$I n_{1}$ & 53 & 75 \\
\hline$I n_{2}$ & 83 & 83 \\
\hline$I n_{3}$ & 74 & 75 \\
\hline
\end{tabular}

Table 2. Configuration parameters for the pedometers.

Therefore, a matrix information was obtained which contains the number of person $I_{i}$, where $i=1,2,3 ; P T_{j}$ the pedometer, where $j=1,2$; the $k$ steps measured

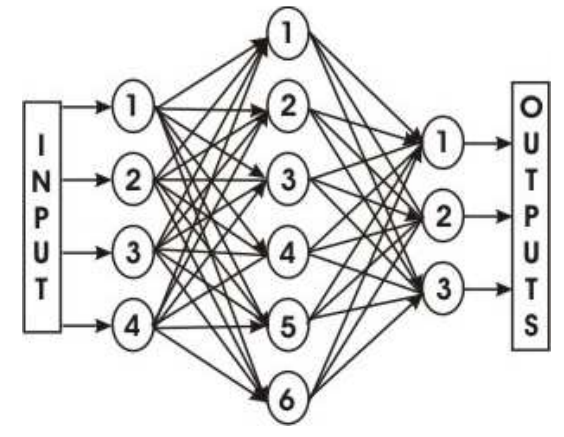

Figure 2. Artificial Neural Network structure applied.

by the $j$ pedometer in the $l$ test is $P_{k, l}^{j}$ and the calories burnt in the same way are $C_{k, l}^{j=2}$. The mean value of the steps and the calories burnt are $\bar{P}_{k, l}^{j}=\frac{1}{10} \sum_{j=1,2} P_{k, l}^{j}$ and $\bar{C}_{k, l}^{j=2}=\frac{1}{10} \sum_{j=2} C_{k, l}^{j}$ respectively in each distance measured for each person $I_{i}$. The $\bar{P}_{k, l}^{j}$ and $\bar{C}_{k, l}^{j=2}$ mensuration are the couple one that allow the construction of the calibration curve used in the next stage.

\subsection{Design Of The Artificial Neural Net- work (ANN)}

The ANN are algorithmic blocks that learn from examples or their input data distribution [6]. Since computers have become more powerful, algorithmic blocks allow the $A N N$ to solve complex problems in many application fields [2]. In this research, in order to synthesize the $A F$ of the pedometer, a progressive $A N N$ [7] has been implemented for three persons. In the experiment, a (4:6:3) structure has been build completely connected with sigmoid activation functions as shown Figure 2, this allows to learn with a single structure the calibration curves obtained in previous stage for the three persons.

\subsection{Design Of The Approximation Func- tion (AF)}

The design of the $A F$ requires an iterative process in order to train the $A N N$, as shows Figure 3 . The training process consists on introducing each double $\left(\bar{P}_{k, l}^{j}, \bar{C}_{k, l}^{j=2}\right)$ to the $A N N$, where the input will be determined by $\bar{P}_{k, l}^{j}$ (number of steps) and the output estimated by $\hat{\bar{C}}_{k, l}^{j=2}$ (calories burnt) that is compared with the real value of calories burn $\bar{C}_{k, l}^{j=2}$ verifying the output error $E=\hat{\bar{C}}_{k, l}^{j=2}-\bar{C}_{k, l}^{j=2}$. The output error is the most important in the training process because it tries to minimize the error applying the iterative 


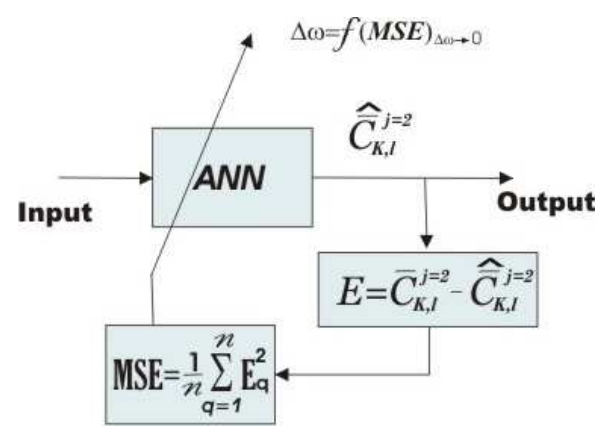

Figure 3. Training process in order to obtain the approximation function minimizing the Mean Squared Error (MSE).

minimization on the $M$ ean $S$ quared $E$ rror $(M S E)$, defined as: $M S E=\frac{1}{n} \sum_{q=1}^{n} E_{q}^{2}=\frac{1}{n} \sum_{q=1}^{n}\left[\hat{\bar{C}}_{k, l}^{j=2}-\bar{C}_{k, l}^{j=2}\right]_{q}^{2}$, where $q$, is the $q$-th element of the calories burn and the estimated calories by the $A N N$. The training algorithm ends when the $M S E$ is minimum, and when this is achieved, it is said that the ANN has learned the calibration curve and it turns into the $A F$ of the pedometer.

\section{Results}

In Figure 4, the results of the calibration curves extracted from 10 exercises applied in three persons between 35 and 350 meters. We can appreciate little differences in the graph of the first person, however, when the step number increases, the calories burn coincide. The graphs in Figure 4 shows the mean values $\left(\bar{P}_{k, l}^{j}\right.$ results, $\left.\bar{C}_{k, l}^{j=2}\right)$ obtained by the pedometer $P T 1$, that it is lower in cost and the pedometer $P T 2$ chosen as the pattern. Obtained the $\left(\bar{P}_{k, l}^{j}, \bar{C}_{k, l}^{j=2}\right)$ values, the $A N N$ was trained in order to learn the synthesized curves that determine the $A F$ of the pedometer, establishing a minimum error $M S E=10^{-4}$ and the structure showed in Figure 2, using a newff function for a Radial Basis Function Networks. Also, the gradient algorithm was applied in order to train the $A N N$ and to synthesize the $A F$ iteratively, the number of epochs were established in 15000. The result of the training is shown in Figure 5., where we can observe that the $M S E$ was achieved in 12135 iterative epochs. Therefore, the $A N N$ has enough knowledge in order to synthesize the $A F$ of the pedometer and to the measures from the three persons.

\section{Discussion}

One of the daily life difficulties in the diabetic patients is the physical exercise accomplishment [1], and the physical activity is showed as a pleasure source that helps in the

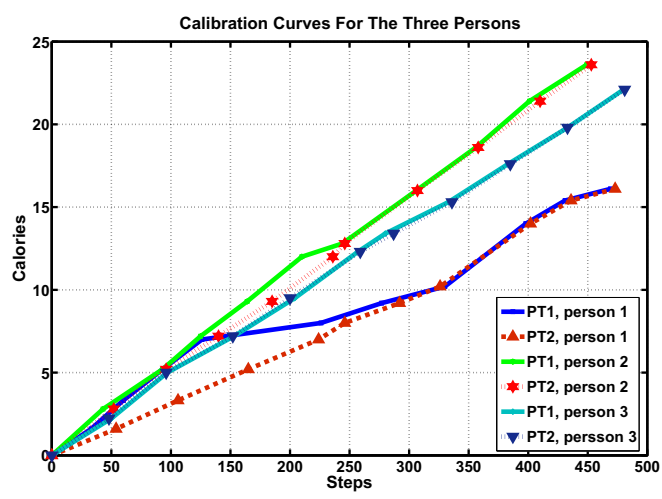

Figure 4. Calibration curves.

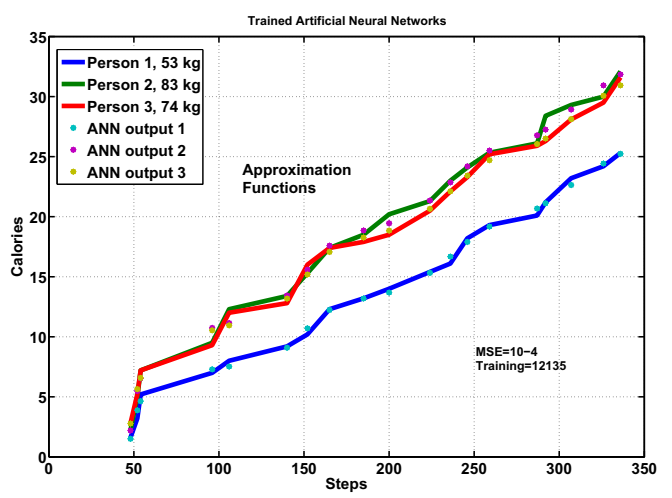

Figure 5. Approximation functions of the pedometers.

weight control and the glucose level in the blood, the persistence of this positive feeling supports the attachment of the patient to the diabetes treatment. In general the patients present low degree of uncertainty regarding to the disease and a high motivation towards the treatment [4]. According to the monitoring development model the treatment includes three phases; 1) the acceptance of the patient with regard to the treatment proposed (that is negotiated among the patient and the health professionals), 2) The Monitoring of the treatment (the patient follows the treatment proposed and his caution health, even if he finds obstacles, and 3) The maintenance of treatment and vigilance of the health (patient takes measures adopted in previous stages with the intention of improving his health and to incorporate them into his life style, transforming the new behaviors in habits. The results in the design of the Approximation Function demonstrate that is possible to train an $A N N$ in order to have reliable data to quantify the physical activity and to arrange information for the adequate insulin supply. 


\section{Conclusions}

- If the information of any pedometer can be validated for a trained $A N N$, there is a possibility that the health professionals can motivate a bigger number of diabetic patients to maintain an adequate level of physical activity, which contributes with the $H L S$.

- The studies demonstrate that the physical activity can develop positive feelings in the diabetic patient towards the treatment in anyone of the three phases.

- The result of this study is the ANN trained, which is the input toward the second phase of this investigation, where the ANN application is approve to support the self-monitoring of the groups of mutual help and its attach to the treatment.

- To use an $A N N$ trained in both diagnosis and clinical interpretation has the following advantages: learning, self-organization, failure tolerance, flexibility and real-time response, the advantages present an extensive range of possibilities for the $A N N$ applications in health sciences as a tool for the diagnosis, and the epidemiological surveillance.

\section{Acknowledgments}

We thank the Computational Intelligence Laboratory (LabINCO) for the help provided to complete this study. LabINCO is a laboratory in the Faculty of Mechanics, Electrics and Electronics Engineering from the University of Guanajuato. Mexico.

\section{References}

[1] Denise Siqueira Péres, Manoel Antônio dos Santos, Maria Lúcia Zanetti and Antônio Augusto Ferronato. Difficulties Of Diabetic Patients In The Illness Control: Feelings And Behaviors. Revista Latino-Americana de Enfermagem, 15:1105 - 1112, November-December 2007.

[2] Diego Andina and Duc Thuong Pham. Computational Intelligence: for Enineering and Manufacturing. Springer-Verlag New York, Inc., Secaucus, NJ, USA, 2007.

[3] Gobierno de los Estados Unidos Mexicanos, Secretaría de Economía, Secretaría de Salud. Norma Oficial Mexicana NOM-015-SSA-1994. In Secretaría de Economía, editor, NOM-015-SSA-1994. Diario Oficial de la Federación, 1994.

[4] João Luís Alves Apóstolo, Catarina Sofia Castro Viveiros, Helena Isabel Ribeiro Nunes and Helena Raquel Faustino Domingues. Illness Uncertainty And Treatment Motivation In Type 2 Diabetes Patients. Revista Latino-Americana de Enfermagem, 15:575-582, 082007.

[5] P. J. Lisboa and Piotr S. Szczepaniak and J. C. Mason and Emmanuel C. Ifeachor. Artificial Neural Networks in Biomedicine. Perspectives in Neural Computing. Springer-Verlag London Berlin Heidelberg, 2000.

[6] Pedro Isasi Viñuela and Inés M. Galván León. Redes de Neuronas Artificiales, Un Enfoque Práctico. Prentice Hall, 2004.

[7] Richard O. Duda, Peter E. Hart and David G. Stork. Pattern Classification. Wiley Interscience Publication, 2001. 